\title{
Conocimiento y actitudes hacia el acomodo razonable de una muestra de la población universitaria de la Universidad de Puerto Rico, Recinto de Río Piedras: Un estudio descriptivo
}

\author{
Knowledge and Attitudes towards Reasonable Accommodation for a Sample of the University \\ Population of Río Piedras Campus at the University of Puerto Rico: A Descriptive Study
}

\section{Conhecimento e atitudes em relação ao alojamento adequado de uma amostra da população universitária na Universidade de Porto Rico, Campus Rio Piedras: Um estudo descritivo}

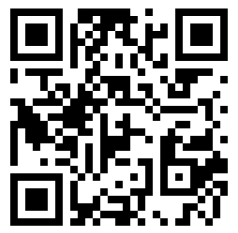

\author{
Lesley Odette Irizarry-Fonseca \\ Universidad de Puerto Rico \\ San Juan, Puerto Rico \\ lesley.irizarry@upr.edu
}

https://orcid.org/0000-0003-3172-3028

\begin{abstract}
Recibido • Received • Recebido: 09 / 07 / 2018
Corregido • Revised • Revisado: 08 / 12 / 2019

Aceptado • Accepted • Aprovado: 13/ 02 / 2020
\end{abstract}

Resumen: El objetivo del estudio fue explorar el conocimiento y las actitudes sobre el acomodo razonable en la población de la Universidad de Puerto Rico, Recinto de Río Piedras. El diseño de la investigación fue descriptivo tipo encuesta, donde participaron 183 estudiantes y sujetos-empleados. Se utilizaron tres instrumentos para explorar datos sociodemográficos, conocimiento sobre acomodo razonable y actitudes. Los datos se analizaron con estadísticas descriptivas de frecuencias y porcentajes. Los hallazgos muestran un nivel de conocimiento moderado y nivel de actitudes favorables en la muestra. No obstante, expresaron deseo de recibir más adiestramientos sobre acomodo razonable y las necesidades particulares de la población con diversidad funcional. Finalmente, se hacen recomendaciones para la planificación curricular y servicios utilizando el diseño universal como base.

Palabras claves: Acomodo razonable; educación postsecundaria; estudiantes con diversidad funcional; diseño universal; diseño curricular.

\begin{abstract}
This study aimed to explore the knowledge and attitudes about reasonable accommodation in the population of Río Piedras Campus at the University of Puerto Rico. The design of the research was a descriptive survey type, where 183 students or employees participated. Three instruments were used to explore sociodemographic data, knowledge about reasonable accommodation and attitudes. The data were analyzed using descriptive statistics of frequencies and percentages. The findings show a moderate level of knowledge and a favorable level of attitudes in the sample. However, they expressed the desire to receive more training on reasonable accommodation and the particular needs of the population with functional diversity. Finally, recommendations are made for curricular planning or services using universal design as a basis.
\end{abstract}

Keywords: Reasonable accommodation; higher education; students with functional diversity; universal design; curricular design. 
http://doi.org/10.15359/ree.24-2.3

ROR: https://ror.org/01t466c14 Universidad Nacional, Costa Rica

http://www.una.ac.cr/educare

educare@una.cr

\begin{abstract}
Resumo: O objetivo do estudo foi explorar conhecimentos e atitudes sobre acomodação adequada na população da Universidade de Porto Rico, Campus Rio Piedras. O desenho da pesquisa foi descritivo e baseado em questionário, envolvendo 183 estudantes e empregados. Foram utilizados três instrumentos para explorar dados sócio demográficos, conhecimentos sobre acomodação adequada e atitudes. Os dados foram analisados com estatísticas descritivas de frequências e porcentagens. Os resultados mostram um nível moderado de conhecimento e nível de atitudes favoráveis na amostra. No entanto, expressaram o desejo de mais treinamento sobre acomodação adequada as necessidades particulares da população com diversidade funcional. Finalmente, são feitas recomendações para o planejamento curricular e serviços usando como base o desenho universal.
\end{abstract}

Palavras-chave: Alojamento razoável; educação universitária; estudantes com diversidade funcional; desenho universal; desenho curricular.

\title{
Introducción
}

Las investigaciones sobre la inclusión, política pública, calidad de viday acomodo razonable de las personas con diversidad funcional han ido en aumento con los años (Bolt et al., 2011; Saleh y Bruyère, 2018). Sin embargo, la población con diversidad funcional continúa siendo excluida y viviendo en condiciones de desventaja en diferentes ámbitos de la vida, particularmente dentro de los escenarios educativos y laborales a nivel mundial (Organización Mundial de la Salud [OMS], 2011). En el escenario laboral, particularmente las personas con impedimentos tienden a estar desempleadas, generar menos ingresos cuando trabajan o estar subutilizadas (Saleh y Bruyère, 2018). En relación con este punto, en el Informe Mundial sobre la Discapacidad se evidencia menor participación laboral entre los varones y mujeres con diversidad funcional con un $53 \%$ y $20 \%$, respectivamente, versus los varones y mujeres sin diversidad funcional con un $65 \%$ y $30 \%$, respectivamente (OMS, 2011). Del mismo modo, el Informe hace referencia a un estudio de la Organización para la Cooperación y el Desarrollo Económicos (OCDE) donde se evidenció en 27 países que las personas con diversidad funcional confrontaban desventajas significativas en el mercado laboral y oportunidades de empleo más pobres (OMS, 2011). Por su parte, Rojas-Rojas et al. (2018) y Schreuer y Sachs (2014) concurren con este planteamiento y añaden que se puede observar una participación menor de la población con diversidad funcional tanto en el escenario laboral como el académico.

Dentro del contexto puertorriqueño, esto se evidencia con las estadísticas recopiladas a través de diferentes fuentes: censo, encuestas especiales, la Oficina de Planificación Académica de la universidad del estado y el Departamento del Trabajo y Recursos Humanos, entre otros. Como dato inicial, es importante establecer que para el año 2016, la población de personas con diversidad funcional, de acuerdo con el estatus de discapacidad en Puerto Rico, representaba el 21.6 por ciento de la población, lo que representa una quinta parte de la población general (Erickson et al., 2018). Para el año 2014 se estimaba que la participación de la población con diversidad funcional en el área laboral era de un $0.5 \%$ del empleo total en la isla (López et al., 2016). Esta cantidad, de acuerdo con la Oficina del Negociado de Estadísticas del Departamento

2

Lesley Odette Irizarry-Fonseca

Los artículos de la Revista Electrónica Educare del Centro de Investigación y Docencia en Educación de la Universidad Nacional, Costa Rica, se comparten bajo términos de la Licencia Creative Commons: Reconocimiento, № Comercial, Sin Obra Derivada 3.0 Costa Rica. Las autorizaciones adicionales a las aquí delimitadas se pueden obtener en el correo: educare@una.cr 
http://doi.org/10.15359/ree.24-2.3

ROR: https://ror.org/01t466c14 Universidad Nacional, Costa Rica http://www.una.ac.cr/educare

del Trabajo, se mantiene, pues no hay nuevos datos que sugieran un aumento (Comunicación Personal, 2018). Al analizar la cifra, a simple vista, es claro que la prevalencia en el mercado laboral es mínima y requiere de un análisis de los factores que contribuyen a esta.

En la Encuesta Especial del Departamento del Trabajo realizada en el 2014, se indicaba que un poco más de la mitad, un $56.3 \%$, de las personas con diversidad funcional habían completado su cuarto año de escuela superior o un año o más de estudios post secundarios (López et al., 2016). Este dato es importante al considerar la interrelación de los cambios en el mercado de empleo y los requisitos de entrada a este como factores que pueden incidir en la empleabilidad y colocabilidad de la población con diversidad funcional en nuestro contexto y el mundo entero. En relación con este planteamiento, Erickson et al. (2018) señalan que aún en la economía actual la educación post secundaria continúa siendo crítica para la obtención de un empleo significativo. En Puerto Rico, para el año académico 2016-2017, la participación en la educación postsecundaria, específicamente en el Recinto de Río Piedras de la Universidad de Puerto Rico, era de un $7.5 \%$ del total de 16872 estudiantes matriculados (UPRRP, Oficina de Planificación Académica, 2017). Este por ciento es similar al de otros recintos e instituciones privadas del país (Báez, 2014). Es evidente que los porcentajes de participación en el sector académico post secundario y el mercado laboral son bajos y que esto puede afectar la calidad de vida de esta población. Particularmente, al significar el empleo como un medio de integración a la sociedad, medio de adquirir bienes y servicios, medio de sustento y promotor de identidad (Saleh y Bruyère, 2018). En reconocimiento de esto, a través de los años se ha desarrollado política pública que promueve la integración y la igualdad de derechos de las personas con diversidad funcional en distintos escenarios y actividades.

Leyes como el Acta de Rehabilitación (1973) y su sección 504, Ley de Prohibición contra el Discrimen Ley 44 (1985), el Acta de Americanos con Discapacidad de 1990 o Ley ADA por sus siglas en inglés (ADA, 1990), protegen los derechos de las personas con diversidad funcional para el disfrute de todas las actividades de la vida, incluyendo las actividades académicas como de empleo en Puerto Rico y Estados Unidos. Uno de los derechos adquiridos es el derecho a solicitar y recibir un acomodo razonable. El acomodo razonable, en el empleo y la educación, pasa a ser un derecho clave, en el ofrecimiento de los servicios óptimos y en la inclusión en sociedad de esta población. Sin embargo, existe mucho desconocimiento sobre los procesos de acomodo razonable. La bibliografía establece la problemática existente en cuanto al entendimiento de la definición de lo que constituye un acomodo razonable (Inniss-Johson, 2012; Unger, 2002). Tanto los entes patronales, el personal educador, como sujetos proveedores de servicios en general, tienden a demostrar falta de conocimiento o entendimiento de lo que esto significa, lo que puede afectar el cumplimiento con la ley y, a su vez, limitar la participación en igualdad de condiciones de las personas con diversidad funcional en distintos escenarios. Por lo antes expuesto, esta investigación tuvo como propósito explorar el conocimiento y actitudes sobre el acomodo razonable en la población universitaria de la Universidad de Puerto Rico, Recinto de Río Piedras. Esto, tomando en consideración la relación entre el conocimiento y las actitudes en el ofrecimiento. 
http://doi.org/10.15359/ree.24-2.3

ROR: https://ror.org/01t466c14 Universidad Nacional, Costa Rica

http://www.una.ac.cr/educare

educare@una.cr

\section{Acomodo razonable: Legislación relacionada, conocimiento y actitudes}

Como se mencionó anteriormente, existen varias leyes a nivel estatal y federal que, a través de los años, han promovido la integración de las personas con diversidad funcional en las distintas actividades del diario vivir en el contexto puertorriqueño. A modo de ejemplo, una pieza legislativa importante en el reconocimiento de los derechos de las personas con diversidad funcional es la ley pública 93-112 conocida como el Acta de Rehabilitación (1973). La sección 504 de esta ley prohíbe la discriminación basada en impedimento en programas y actividades públicas y privadas que reciben fondos federales. A nivel estatal se encuentra la Ley 44 (1985) conocida como Ley que Prohíbe el Discrimen contra las Personas con Impedimentos. Esta Ley, entre otros aspectos, contiene disposiciones sobre el derecho a acomodo razonable, definido en su Artículo 1:

El ajuste lógico adecuado o razonable que permite o faculta a una persona cualificada para el trabajo, con limitaciones físicas, mentales o sensoriales ejecutar o desempeñar las labores asignadas a una descripción o definición ocupacional ... y que no representa un esfuerzo extremadamente oneroso en términos económicos ... (p. 1)

Además, establece que la persona tiene derecho a la participación e integración en la sociedad a través de la adaptación, modificación o medida de ajuste en las áreas de empleo, educación, transportación, vivienda, entre otras (Ley 44, 1985). Específicamente, en el artículo 10 de la enmienda de 1991, se establece que las instituciones que ofrezcan servicios de enseñanza no podrán discriminar contra las personas con impedimentos cualificados. En particular, responsabiliza a la Universidad de Puerto Rico a capacitar profesionales que brinden servicio a las personas con impedimentos de acuerdo con la demanda por estos servicios (Oliveras, 2012). No obstante, no es sino hasta 1990, cuando se aprueba la Ley de Americanos con Discapacidad, conocida como ley ADA por sus siglas en inglés, que se comienza a observar un aumento en la participación de las personas con diversidad funcional en las actividades del diario vivir, aunque aún se considera baja en comparación con la población en general (Rojas-Rojas et al., 2018; Saleh y Bruyère, 2018). La Ley ADA (1990) es una pieza de derechos civiles donde se prohíbe el discrimen contra las personas con impedimentos o diversidad funcional, se aplica a distintos sectores, no solo a aquellos que reciban fondos federales. Contiene 5 Títulos. En particular, el Título II establece que todas las agencias locales y estatales, servicios, programas y actividades no deben discriminar en contra de las personas con impedimentos o diversidad funcional. Entre las entidades o servicios obligados bajo este Título se encuentran las instituciones educativas del gobierno, incluyendo universidades y escuelas elementales y secundarias.

Existen otras leyes a nivel estatal que también velan por los derechos y la integración de las personas con diversidad funcional. Una de ellas es la Ley 51 (1996), Ley de Servicios Educativos Integrales para Personas con Impedimentos. Esta, en su Artículo 2, define un acomodo razonable 
http://doi.org/10.15359/ree.24-2.3

ROR: https://ror.org/01t466c14 Universidad Nacional, Costa Rica http://www.una.ac.cr/educare

como la "modificación o ajuste al proceso o escenario educativo o de trabajo que permita a la persona con impedimentos participar y desempeñarse en ese ambiente..." (párr. 5). La Ley 51 (1996) también establece responsabilidades para la Universidad de Puerto Rico. Por su parte, la Ley 238 (2004) conocida como la Carta de Derechos de las personas con impedimento tiene el propósito de garantizar mejor calidad de vida para las personas con diversidad funcional a través de la inclusión en todas las áreas como empleo, educación, transportación, recreación, seguridad, entre otras (Ley 238, 2004). Ahora bien, aunque existen diferentes mandatos de ley sobre el acomodo razonable, la bibliografía establece que aún existe confusión y desconocimiento sobre las leyes y sobre lo que es un acomodo razonable. En las investigaciones se ha encontrado que la clase patronal presenta un conocimiento entre moderado a bajo sobre aspectos relacionados a la Ley ADA (Inniss-Johnson, 2012; Unger, 2002). Más aún, no tienen un conocimiento claro sobre su rol en la implementación de la ley (Hernandez et al., 2000; InnissJohnson, 2012; Unger, 2002). Sin embargo, se considera que el éxito de dicha implementación depende, en gran medida, del conocimiento sobre la ley (Hernandez et al., 2000).

Por su parte, investigaciones realizadas sobre acomodos en los escenarios académicos y la relación con el conocimiento han presentado resultados similares. Uno de estos estudios fue realizado por Rao y Gartin (2003). En el estudio se exploraba la actitud de la facultad universitaria con respecto a los acomodos brindados a estudiantes con impedimentos o diversidad funcional. Los autores encontraron que la facultad a la que pertenecía el profesor o profesora; las experiencias previas con personas con impedimento y el conocimiento sobre las leyes influía significativamente en la disposición de estos para honrar o brindar los acomodos razonables. Estos hallazgos son importantes, pues se considera que el conocimiento es una variable que impacta las actitudes (Innis-Johnson, 2012). Las actitudes juegan un papel importante a la hora de evaluar los obstáculos que presenta la población de personas con diversidad funcional en la sociedad. A pesar de la identificación de otras barreras, las barreras actitudinales continúan siendo uno de los impedimentos más significativos para la plena inclusión y participación de las personas con diversidad funcional en los diferentes ámbitos de la vida (OMS, 2011; Saleh y Bruyère, 2018; Unger, 2002). Se ha evidenciado que las actitudes favorables hacia las personas con diversidad funcional y un trato de igualdad puede llevar a una actitud favorable sobre el acomodo razonable en los distintos escenarios, promoviendo la inclusión de las personas con diversidad funcional (Innis-Johnson, 2012). Por lo antes expuesto, el propósito del estudio no se limitó a explorar el conocimiento, sino también las actitudes sobre el acomodo razonable en el escenario universitario. Esto, tomando en consideración la relación entre el conocimiento y las actitudes en el ofrecimiento de los acomodos. 
http://doi.org/10.15359/ree.24-2.3

ROR: https://ror.org/01t466c14 Universidad Nacional, Costa Rica

http://www.una.ac.cr/educare

educare@una.cr

\section{Metodología}

\section{Diseño}

Para llevar a cabo el estudio se utilizó un diseño de investigación descriptivo tipo encuesta. Se utilizaron estadísticas descriptivas como frecuencias y por cientos para el análisis de los datos.

\section{Participantes}

La muestra del estudio fue seleccionada por disponibilidad. Estuvo constituida por 183 estudiantes de universidad. El grupo participante se define como estudiantes o persona empleada de la Universidad de Puerto Rico, Recinto de Río Piedras. El único criterio de inclusión de las personas participantes fue ser empleada o estudiante de la Universidad de Puerto Rico, Recinto de Río Piedras (UPR-RP).

\section{Instrumentos}

Para el estudio se utilizaron tres instrumentos creados por Concepción y Rodríguez (1998), modificados por Oliveras (2012). El primero fue una planilla de datos sociodemográficos que contiene 13 preguntas que recopilaba la información descriptiva sobre edad, género, facultad, grado académico más alto, entre otros. El segundo instrumento era un cuestionario de 12 premisas que exploraban el grado de conocimiento sobre los aspectos legales de acomodo razonable. El tercer instrumento era una escala que exploraba actitud hacia cuán dispuesto está el sujeto participante a otorgar el acomodo razonable, cuán necesario son los acomodos, cuánto afecta la dinámica en el salón de clases y cuán justo es el acomodo con respecto a los compañeros y compañeras de clase. Este mismo consistía de 32 premisas que se contestaban con una escala Likert, de 4 puntos, que fluctuaba de totalmente de acuerdo a totalmente en desacuerdo.

\section{Procedimiento}

El primer paso consistió de la solicitud para llevar a cabo la investigación al Comité Institucional para la Protección de los Seres Humanos en la Investigación (CIPSHI) de la Universidad de Puerto Rico, Recinto de Río Piedras. Una vez se obtuvo la autorización, se envió la carta para el reclutamiento de las personas participantes y administración del cuestionario a través del correo institucional. El correo electrónico enviado a la comunidad universitaria contenía el enlace a la encuesta a través de la herramienta digital de SurveyMonkey. Este correo electrónico también contenía el consentimiento informado como un documento aparte para la revisión y copia de cada participante. Al accesar el enlace en el correo electrónico cada participante encontraría el consentimiento informado nuevamente. Al presionar el botón de ACEPTO se entendía que había leído el consentimiento y estaba de acuerdo en participar, no obstante, podía cesar su participación en cualquier momento de desearlo así.

6 
http://doi.org/10.15359/ree.24-2.3

ROR: https://ror.org/01t466c14 Universidad Nacional, Costa Rica http://www.una.ac.cr/educare

educare@una.cr

El acceso al instrumento a través del enlace de SurveyMonkey estuvo disponible por un periodo de dos meses, luego del primer correo electrónico enviado. Se enviaron dos correos electrónicos. El primero contenía la invitación a participar, junto a la Hoja de consentimiento y el enlace a los instrumentos. El segundo correo luego de transcurrido un mes del primer correo, se envió como un recordatorio de la investigación. Al cabo de los dos meses se cerró el acceso al enlace y se procedió a la tabulación de los datos utilizando el programa de SurveyMonkey.

\section{Resultados, análisis e interpretación}

\section{Datos sociodemográficos}

El estudio contó con un total de 183 participantes de forma voluntaria. Sexo: El $75.57 \%$ $(n=134)$ de participantes indicó ser del género femenino y $24.43 \%(n=44)$ de género masculino. Seis participantes no reportaron el sexo. Rango de edad: Las edades de las personas participantes se encontraban en los siguientes rangos de edad: el $22.60 \%(n=40)$ indicó tener 21 años o menos, el $27.60 \%(n=49)$ de $21-30$ años, el $9.60 \%(n=17)$ de $31-40$ años, el $17.50 \%(n=31)$ de $41-50$ años, el $16.38 \%(n=29)$ indicó que tenía $51-60$ años, el $6.15 \%(n=11)$ con 61 años o más, 5 participantes no respondieron la pregunta. Grado académico más alto: En cuanto al nivel más alto de escolaridad alcanzado, el $32.40 \%(n=58)$ expresó haber culminado la escuela superior, el 20.90 $\%(n=37)$ su bachillerato, un $23.16 \%(n=41)$ su maestría, el $19.21 \%(n=34)$ un grado doctoral y el $3.95 \%(n=7)$ otros, entre los cuales destacaron un grado asociado. Rol del participante: Al explorar el rol de cada participante en la universidad, se encontró que la mayoría fue estudiantes, representando el $61.45 \%(n=110)$ de quienes participaron. Un $20.67 \%(n=37)$ indicó ser docente, un $7.82 \%(n=14)$ se identificó como personal no docente - servicio al estudiantado, $13.97 \%(n=25)$ personal no docente - otro, y un 7.82 \% expresó estar en otra área. Tiempo en la universidad: Se exploró, además, el tiempo que lleva el sujeto participante en la universidad como personal empleado o estudiante. El $49.72 \%(n=89)$ indicó que lleva de $0-5$ años estudiando o trabajando en la universidad, el $20.67 \%$ ( $n=37)$ de 6-11 años. Esto es congruente con el rol de estudiante de la mayor parte de las personas participantes, según indicado en la premisa anterior. Por su parte, un $7.26 \%(n=13)$ indicó que llevan de 12-17 años y 18-23 años respectivamente, el $7.82 \%(n=14)$ de 24-29 años y otro $7.26 \%$ ( $n=13)$ más de 30 años en la universidad. Facultad: En cuanto a la facultad a la que pertenecían, la Facultad de Ciencias Sociales y Educación fueron las facultades con mayor número de participantes con un $16.97 \%(n=28)$ respectivamente. Un $14.55 \%(n=24)$ indicó pertenecer a las Facultades de Humanidades y Ciencias Naturales, respectivamente. El $6.67 \%(n=11)$ está adscrito a la Facultad de Administración de Empresas y $4.85 \%(n=8)$ está en Estudios Generales. Por su parte, la Escuela de Derecho y la Escuela de Comunicaciones tuvieron una representación de un $4.24 \%(n=7)$, respectivamente. La Escuela de Arquitectura tuvo un $3.03 \%(n=5)$ y la escuela Graduada de Planificación un $0.61 \%(n=1)$. El $13.50 \%(n=22)$ restante de quienes participaron marcaron que estaban en otras áreas que incluían: biblioteca, administración y rectoría. Solicitud de acomodo razonable: Al explorar si ha recibido algún 
http://doi.org/10.15359/ree.24-2.3

ROR: https://ror.org/01t466c14 Universidad Nacional, Costa Rica

http://www.una.ac.cr/educare

educare@una.cr

estudiante o personal con una solicitud de acomodo razonable, el $37.80 \%(n=62)$ indicó haber recibido solicitudes, el $12.80 \%(n=21)$ que no han recibido solicitudes y el $49.39 \%(n=81)$ que no le aplicaba. También se exploró si la persona participante había solicitado acomodo razonable. El $21.82 \%(n=36)$ indicó que ha solicitado acomodo razonable versus un $78.18 \%(n=129)$ que no lo había solicitado. Acomodo solicitado: A las personas participantes que indicaron haber solicitado acomodo razonable se les pidió que indicaran cuál fue el acomodo solicitado de acuerdo con las alternativas presentadas. El acomodo solicitado con mayor frecuencia fue tiempo adicional con un $22.79 \%(n=31)$. Un $4.41 \%(n=6)$ solicitó el acomodo de equipo asistivo (AT), un $1.47 \%(n=2)$ solicitó el uso de asistente y un $0.74 \%(n=1)$ solicitó el uso de grabadora. Ninguna de las personas participantes indicó haber solicitado el uso de intérprete y el $16.91 \%(n=23)$ restante indicó que solicitó otro acomodo razonable. Ejemplos de otros acomodos solicitados son: reubicación de un salón de clases, un estacionamiento más cercano, tareas fragmentadas, recesos y cambio de puesto, entre otros. Esto es consistente con los resultados de un estudio realizado por Bolt et al. (2011), donde encontraron que los tres acomodos más utilizados en el escenario universitario fueron: tiempo adicional, ajuste en el escenario, y equipo de asistencia tecnológica, específicamente lector de pantalla, y consideraron estos de mucha ayuda. Por otra parte, el $18.90 \%(n=24)$ de los sujetos encuestados que solicitaron acomodos indicó que tuvo dificultad para proveer o recibir el acomodo razonable y el $81.10 \%(n=103)$ que no presentaron dificultad. Dificultad al solicitar o recibir el acomodo razonable: A las personas participantes que indicaron que habían confrontado dificultad para recibir u otorgar el acomodo razonable, se les solicitó que indicaran a qué se debía la dificultad. Al analizar las respuestas las mismas se categorizaron en 4 áreas principales. Tipo de acomodo ofrecido; actitudes; adiestramiento/ conocimiento; y aspectos administrativos. Algunos ejemplos de las respuestas de las personas participantes en cuanto a las dificultades con el tipo de acomodo son los siguientes: modificación de módulo de forma apropiada de acuerdo con las necesidades del empleado (participante 27), el tiempo dado del profesor no fue el correcto para mí (participante 22). Este aspecto esencial en la recomendación de acomodos no ha sido estudiado con la frecuencia necesaria. De hecho, la bibliografía se ha enfocado más en la legislación y la implementación de esta, explorando con menor frecuencia la utilidad de los acomodos razonables para la persona con diversidad funcional (Schreuer y Sachs, 2014). Más aún, se establece que todavía existe desconocimiento sobre el proceso interactivo para la recomendación de acomodo razonable, el mal manejo del acomodo razonable representa un $25 \%$ de las querellas ante la Comisión de Igualdad de Oportunidades en el Empleo (EEOC por sus siglas en inglés) (Gold et al., 2012).

En cuanto a la segunda categoría de actitudes, las personas participantes expresaron lo siguiente: las profesoras manifiestan que, si no se puede realizar las tareas sin el acomodo, entonces uno escogió la profesión equivocada (participante 23). Estas expresiones son cónsonas con los hallazgos de Timmerman y Mulvihill (2015), donde establecen que el divulgar un impedimento o solicitud de acomodo razonable también puede ser discriminatorio por la percepción o creencia de que las personas no sean capaces. Otros sujetos participantes indicaron que: No es honrado

8

Lesley Odette Irizarry-Fonseca

Los artículos de la Revista Electrónica Educare del Centro de Investigación y Docencia en Educación de la Universidad Nacional, Costa Rica, se comparten bajo términos de la Licencia Creative Commons: Reconocimiento, № Comercial, Sin Obra Derivada 3.0 Costa Rica. Las autorizaciones adicionales a las aquí delimitadas se pueden obtener en el correo: educare@una.cr 
http://doi.org/10.15359/ree.24-2.3

ROR: https://ror.org/01t466c14 Universidad Nacional, Costa Rica http://www.una.ac.cr/educare

por las facultades y muchos de los profesores (participante 15), personal poco amable y no empático (participante 07), A pesar de la carta de acomodo solicité el uso de grabadora y se me negó, pues a los profesores no les gusta ... (participante 09). Estas respuestas sugieren que a pesar de la existencia de leyes como el Acta de Rehabilitación (1973), que establece que no se le puede negar el uso de equipo de asistencia tecnológica como una grabadora a la persona con impedimento. Estas son las experiencias de algunas de las personas participantes, lo cual evidencia que no es la existencia de las leyes sino las actitudes o el desconocimiento lo que provoca que no se cumplan o se provea el trato igualitario a las personas con diversidad funcional.

En la tercera área identificada relacionada a conocimiento/adiestramientos sobre acomodo razonable, las respuestas de las personas participantes reflejaron dudas y falta de conocimiento tanto del estudiantado como del profesorado y del personal empleado sobre el acomodo razonable. Algunas de las expresiones fueron las siguientes: ... no hemos recibido adiestramiento sobre la política institucional y nuestra responsabilidad funcional ni legal como empleados expresó el participante 22. Por su parte, el participante 21 expresó que: Varios profesores no entendían cómo cumplir con el listado de acomodos que estipulaba la carta de OAPI. Estas expresiones son similares a las del participante 09 que indicó que el personal no sabía cómo ayudarlo. Además, otro participante indicó ... otros profesores entienden que darme tiempo adicional es darme ventaja sobre los demás (participante 02). A través de las respuestas se puede confirmar la existencia de los servicios a las personas con diversidad funcional a través de Oficina de Asuntos a Personas con Impedimento (OAPI) del Recinto de Río Piedras, con lo cual se cumple con la política pública. Sin embargo, aunque la universidad cumple con esta política, principalmente, el personal docente no se siente preparado. Hay que tomar en consideración que no todos los programas académicos trabajan en su currículo la diversidad y otros piensan que ofrecen lo necesario. Por otra parte, las expresiones también son cónsonas con lo encontrado en un estudio reciente de Schwartz (2016), quien exploró el conocimiento sobre lo que es acomodo razonable. Encontró que mientras estudiantes entendían que no estaban recibiendo un acomodo razonable, el personal pensaba que sí lo estaba ofreciendo.

La última categoría establecida está relacionada con los aspectos administrativos: Entre las verbalizaciones se encuentran: TIEMPODE: solicitud, cita, evaluación, aprobación, reintegración (participante 27); A pesar de la carta a la Rectora y una reunión, luego de su renuncia no se ha hecho nada (participante 11); La estudiante indica que quería tomar el examen en otro salón alejada de otras personas y yo no tenía el personal suficiente para hacerlo a la misma vez que daba el examen regular (participante 02). Estas expresiones concurren con hallazgos encontrados en estudios como el de Bolt et al. (2011). En este estudio el equipo investigador exploró las barreras percibidas por estudiantes sobre los acomodos que recibían o el proceso de solicitud. Las mayores barreras encontradas estaban relacionadas con el sistema, como: falta de dinero, problemas con el proceso de otorgación de acomodos específicos o la falta de estos. 
http://doi.org/10.15359/ree.24-2.3

ROR: https://ror.org/01t466c14 Universidad Nacional, Costa Rica

http://www.una.ac.cr/educare

educare@una.cr

Adiestramientos recibidos: La última pregunta del instrumento de información sociodemográfica y general exploraba, si habían recibido algún adiestramiento, taller, conferencia, seminario o si había realizado lecturas sobre alguna de las leyes que se mencionaban: Ley ADA (1990) según enmendada, Ley 51 (1996) según enmendada, Ley 44 (1985) según enmendada, Ley 238 (2004), u otra ley. Entre los adiestramientos de leyes prevalece la Ley ADA (1990) según enmendada con un $75.76 \%$ ( $n=75)$, la Ley 238 (2004) con un $62.63 \%(n=62)$, y la Ley 51 (1996) con un 53.54 $\%(n=53)$. Las últimas dos leyes mencionadas tienen mandatos específicos para la capacitación de personal. Además, un $9.09 \%$ indicó haber recibido adiestramientos sobre otras leyes.

\section{Exploración de conocimiento}

El segundo instrumento exploraba el conocimiento de las personas participantes sobre el acomodo razonable. Lo primero que se preguntó fue de qué índole es el acomodo razonable. La mayoría de quienes participaron entienden que es un aspecto legal con un $83.19 \%$ ( $n=99$ ). El $8.40 \%(n=10)$ indicó que es un aspecto de índole académico, el $3.36 \%(n=4)$ entiende que es de índole ético y el $5.04 \%(n=6)$ restante entiende que es de índole social. Luego se presentaron varias definiciones de acomodo razonable donde cada participante debía seleccionar la mejor alternativa. Dentro de las definiciones ofrecidas de acomodo razonable, la mayor parte (89.2\%) seleccionaron un ajuste o modificaciones que le garantizan igualdad de posibilidades a las personas con impedimentos, debidamente cualificada, para realizar las funciones esenciales del puesto, como la definición de lo que es un acomodo razonable. Por otra parte, otras definiciones se presentan en la Tabla 1. Estas evidencian que aún existe confusión en cuanto a lo que es un acomodo.

Tabla 1: Distribución de frecuencias y por cientos por definición

\begin{tabular}{lc}
\hline \multicolumn{1}{c}{ Premisa } & $\mathrm{n}$ \\
\hline $\begin{array}{l}\text { Ajuste o modificaciones que le garantizan igualdad de posibilidades a las personas con } \\
\text { impedimentos, debidamente cualificada, para realizar las funciones esenciales del puesto. }\end{array}$ & 110 \\
\hline $\begin{array}{l}\text { Ajuste o modificaciones que le garantizan a la persona con impedimentos realizar las funciones } \\
\text { esenciales de su puesto, aunque no esté debidamente cualificada }\end{array}$ & 7 \\
$\begin{array}{l}\text { Privilegios que obligan al ente patronal a realizar aquellas modificaciones necesarias para las } \\
\text { personas con impedimentos }\end{array}$ & 4.7 \\
$\begin{array}{l}\text { Concepto ético-moral que promueve la inclusión de la persona con impedimentos en un } \\
\text { trabajo u ocupación }\end{array}$ & 3.3 \\
\hline
\end{tabular}

Nota: Elaboración propia.

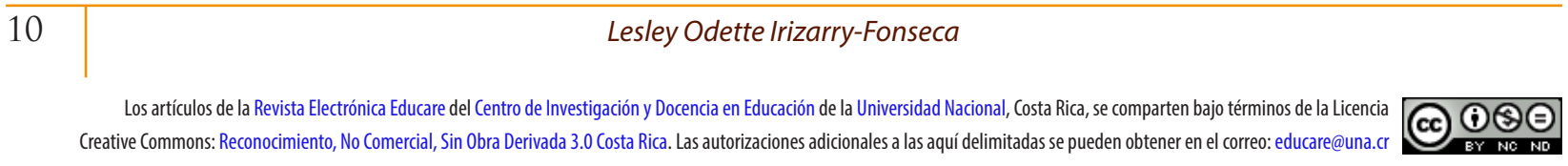


http://doi.org/10.15359/ree.24-2.3 ROR: https://ror.org/01t466c14 Universidad Nacional, Costa Rica http://www.una.ac.cr/educare

La tercera premisa exploraba conocimiento de las personas participantes sobre la sección 504 de la Ley de Rehabilitación (Acta de Rehabilitación, 1973). Específicamente se les preguntó qué establece dicha sección y las opciones eran las siguientes: ninguna institución pública o privada, que reciba fondos estatales podrá discriminar contra una persona debidamente cualificada por razón de este impedimento que obtuvo el $14.56 \%(n=15)$ de las respuestas; ninguna institución pública o privada, que reciba fondos del gobierno de $P R$, podrá discriminar contra una persona debidamente cualificada por razón de este impedimento que fue seleccionada por el $19.42 \%(\mathrm{n}=20)$ de las personas participantes; ninguna institución pública o privada, que reciba fondos municipales, podrá discriminar contra una persona debidamente cualificada por razón de este impedimento que no fue seleccionada por ningún participante; y ninguna institución pública o privada, que reciba fondos federales, podrá discriminar contra una persona debidamente cualificada por razón de este impedimento que obtuvo la mayoría de las respuestas con un $66.02 \%(n=68)$. Sin embargo, hubo mayor variabilidad en las respuestas de las personas participantes, lo que puede ser congruente con el porcentaje menor que indicó haber recibido adiestramientos y talleres, entre otros, sobre el Acta de Rehabilitación (1973). En la siguiente premisa se puede observar, de igual manera, mayor variabilidad entre las respuestas seleccionadas. En esta ocasión se le solicitó a quienes participaron que indicaran cuál de las siguientes aseveraciones era falsa con respecto al Acta de Rehabilitación: la primera aseveración era Tratar de estimular a las instituciones públicas y privadas que reciben fondos federales a que brinden un trato justo a la persona con impedimento. Esta alternativa fue seleccionada por el $10.48 \%(n=11)$ de las personas participantes. La segunda opción era Dispone que las instituciones públicas y privadas que reciban fondos federales deberán establecer sistemas de cuotas para garantizar la participación igualitaria a de personas con impedimentos en el ámbito educativo y ocupacional que obtuvo el $60.95 \%(n=64)$ de las respuestas. La tercera aseveración era Obliga a efectuar los ajustes académicos necesarios para no obstaculizar los objetos educativos de los estudiantes universitarios con impedimentos, que obtuvo el $13.33 \%(n=14)$ de las respuestas. La última aseveración Prohíbe establecer normas y criterios cuyo efecto sea discriminar contra la persona con impedimentos cualificada, obtuvo un $15.24 \%(n=16)$ de las respuestas.

La siguiente pregunta leía: Según la ley $A D A$, se le requiere hacer un acomodo razonable a una entidad pública o privada que este cubierta por la ley cuando: La primera opción era: la persona con impedimentos debidamente cualificada así lo solicite, que obtuvo un $76.15 \%$ ( $\mathrm{n}=83$ ) de las respuestas; la segunda opción indicaba: este represente un costo oneroso al patrono, agencia o institución, que obtuvo $1.83 \%(n=2)$ de las respuestas; la tercera aseveración era la persona con impedimentos no esté cualificada para cumplir con las funciones esenciales de un trabajo u ocupación, que obtuvo un $0.92 \%(n=1)$ y la cuarta aseveración era siempre, aun cuando la persona con impedimento debidamente cualificada no lo solicite, que obtuvo un 21.10 $\%(\mathrm{n}=23)$ de las respuestas. Del mismo modo se le preguntó a cada participante lo siguiente: La ley ADA incorpora el concepto de acomodo razonable, el cual implica que entidad pública o privada cubierta por la ley debe: Entre las alternativas presentadas, al igual que en la premisa anterior, la mayor parte de las personas participantes seleccionaron la mejor respuesta. El 77.67 
http://doi.org/10.15359/ree.24-2.3

ROR: https://ror.org/01t466c14 Universidad Nacional, Costa Rica

http://www.una.ac.cr/educare

educare@una.cr

$\%(n=80)$ seleccionó la respuesta realiza los ajustes necesarios para garantizar que la persona con impedimentos debidamente cualificada tenga los mismos derechos que la persona que no tiene impedimentos. La próxima respuesta con mayor porcentaje fue establece un estándar de calidad o cantidad que le permita a la persona con impedimentos debidamente cualificada ejecutar sus tareas, la cual obtuvo un $10.68 \%(n=11)$. Las otras respuestas fueron haga la inversión necesaria, no importa el costo, para cumplir con las disposiciones de la ley y otorga privilegios a la persona con impedimentos debidamente cualificada para que pueda participar en los programas o actividades que ofrece; las cuales alcanzaron un $6.80 \%(n=7)$ y $4.85 \%(n=5)$, respectivamente.

Sobre la premisa número siete se le presentaron varios escenarios a las personas participantes para que seleccionaran cuál de estos correspondía a un escenario de acomodo razonable.: Las opciones a elegir eran: Eximir al estudiante de tomar exámenes para su evaluación académica y Permitir que el estudiante conteste sus exámenes sin límite de tiempo alguno que obtuvieron un $5.26 \%(n=6)$ de las respuestas, respectivamente. Permitir el uso de anotadores, lectores y la adquisición de equipos tecnológicos o asistivos que le permitan tener igual oportunidad de aprendizaje al estudiante, fue seleccionada por la mayor parte de las personas participantes con un $85.96 \%$ ( $\mathrm{n=98)}$. Por último, la implantación de un estándar mínimo de calidad y cantidad en los cursos académicos en los que se matricule el estudiante, fue seleccionada por el $3.54 \%$ ( $n=4)$ de los sujetos encuestados. Al momento de explorar si podían identificar cuál de las opciones sería una violación a la ley ADA, la aseveración con más puntuación fue imponer a la persona con impedimentos debidamente cualificada que acepte un acomodo razonable determinado, que obtuvo un $52.88 \%(n=55)$. Es importante destacar que en el proceso interactivo de evaluación para la recomendación de un acomodo razonable siempre se debe contar con la participación de la persona solicitante del acomodo. Por otra parte, la segunda opción era establecer que la persona con impedimentos debidamente cualificada puede llevar a cabo las funciones esenciales de un trabajo u ocupación con o sin acomodo. Esta opción obtuvo un 30.48 \%, sugiere, así, desconocimiento sobre la definición de persona cualificada con impedimento en la Ley ADA (1990). Las siguientes alternativas obtuvieron porcentajes más bajos: ofrece información de la ley ADA en los medios de comunicación interna y externa del patrono, agencia o institución y provee a la persona con impedimentos debidamente cualificada la oportunidad de recibir servicios y participar de forma integrada en programas, servicios y actividades que se ofrecen en la comunidad en general, puntuaron $8.65 \%(n=9)$ y $7.69 \%(n=8)$, respectivamente.

Luego se exploró el conocimiento sobre los factores que se consideran para determinar si el acomodo es una carga onerosa para el sector patronal. Las alternativas eran: El nivel de producción de la persona con impedimento, que obtuvo $11.21 \%(n=12)$; La naturaleza y el costo del acomodo razonable que obtuvo un $66.36 \%(n=71)$; La situación social y económica de la persona con impedimento que obtuvo $1.87 \%(\mathrm{n}=2)$; y la Eficiencia de la persona con impedimento en el desempeño de sus funciones que obtuvo un $20.56 \%(n=22)$. De la siguiente aseveración, las personas participantes tenían que seleccionar la alternativa que entendían representaba un

\begin{tabular}{l|l}
\hline 12 & Lesley Odette Irizarry-Fonseca
\end{tabular}

Los artículos de la Revista Electrónica Educare del Centro de Investigación y Docencia en Educación de la Universidad Nacional, Costa Rica, se comparten bajo términos de la Licencia Creative Commons: Reconocimiento, No Comercial, Sin Obra Derivada 3.0 Costa Rica. Las autorizaciones adicionales a las aquí delimitadas se pueden obtener en el correo: educare@una.cr 
http://doi.org/10.15359/ree.24-2.3

ROR: https://ror.org/01t466c14 Universidad Nacional, Costa Rica http://www.una.ac.cr/educare

educare@una.cr

acomodo razonable para estudiantes en un curso. Las alternativas fueron las siguientes: Preparar tareas con un grado mínimo de dificultad, con un $1.80 \%(n=2)$. Exigir a los demás estudiantes que provean las anotaciones de la clase, la cual fue seleccionada por el $3.60 \%(n=4)$ de quienes participaron. Eximir a los estudiantes de tomar exámenes, que fue seleccionada por el $1.80 \%$ $(n=2)$ de las personas participantes. Por último, Darle tiempo adicional en los exámenes, la cual obtuvo un $92.79 \%(n=103)$ y fue la que puntuó más alto dentro de las opciones brindadas. En las últimas dos preguntas del cuestionario, se buscaba explorar el conocimiento sobre la Ley 51 (1996), donde, en primer lugar, tenían que completar la oración toda persona con impedimento tendrá derecho a: la primera opción era Seleccionar, diseñar, ajustar, adaptar, hacer a la medida, aplicar, mantener, reparar o reemplazar equipo de asistencia tecnológica, la cual obtuvo el 8.00 $\%(n=8)$. La segunda opción Recibir los servicios integrales que responden a sus necesidades particulares fue seleccionada por el $78.00 \%(n=78)$ de las personas participantes. La tercera opción fue Decidir por sí mismo, de acuerdo a su condición clínica, para escoger entre diferentes opciones que le sean planteadas con un $11.00 \%(n=11)$ de las personas participantes, y la última opción, Participar de las actividades y programas de autosuficiencia de su apoyo en la comunidad fue seleccionada por el $3.03 \%(n=3)$ de las personas que contestaron la pregunta. La segunda pregunta sobre la Ley 51 (1996) decía: Bajo la ley 51, la Universidad de PR tiene la responsabilidad de. Las opciones para escoger eran las siguientes: Proveer servicios congruentes y comprensivos de acuerdo con la condición; Asumir responsabilidad sobre la recuperación del estudiante y fomentar la responsabilidad de este; Capacitar a un número razonable de profesionales que brinden servicios a las personas con impedimentos de acuerdo con la demanda por estos servicios; y Graduar al estudiante, aunque no esté debidamente cualificado. Las premisas mencionadas obtuvieron en puntuación: $66.99 \%(n=69), 2.91 \%(n=3), 28.16 \%(n=29)$ y $1.94 \%(n=2)$, respectivamente.

A través de las respuestas ofrecidas se puede observar mayor variabilidad en las respuestas en las premisas que no están relacionadas a la Ley ADA de 1990. Esto puede responder a que un porcentaje mayor de participantes había identificado previamente haber recibido adiestramientos sobre las piezas legislativas: Ley ADA y Carta de Derechos de 2004. Esta falta de conocimiento sobre la legislación vigente dentro de los escenarios educativos y laborales puede incidir en las actitudes que se presentan sobre la población y los acomodos razonables. Esto se ilustra en un estudio realizado por Basilice (2015) sobre la relación entre conocimiento y actitudes con la capacidad para apoyar a estudiantes con diversidad funcional de un profesorado universitario donde se encontró que este grupo reconocía tener falta de conocimiento y capacidad limitada para ayudar a sus estudiantes de manera efectiva, independientemente de su desarrollo profesional.

\section{Exploración de actitudes}

De acuerdo con la bibliografía, las actitudes negativas representan una barrera para las personas con diversidad funcional (Copeland et al., 2010; OMS, 2011; Timmerman y Mulvihill, 
http://doi.org/10.15359/ree.24-2.3

ROR: https://ror.org/01t466c14 Universidad Nacional, Costa Rica

http://www.una.ac.cr/educare

educare@una.cr

2015). Sin embargo, actitudes favorables pueden facilitar su inclusión en las distintas actividades del diario vivir y promover el acomodo razonable. A tales efectos, el tercer instrumento utilizado tenía el propósito de explorar las actitudes de las personas participantes sobre el acomodo razonable. Tenían que expresar cuán de acuerdo o en desacuerdo estaban con las premisas presentadas utilizando una escala Likert de cuatro puntos. Las premisas estaban presentadas en una dirección positiva y otras en negativo, por lo que para analizar las puntuaciones se invirtió la dirección de las que así lo ameritaban y se calcularon los promedios. Las puntuaciones obtenidas por la muestra fluctuaron entre 2.70 a 3.87. Se consideró una actitud sumamente favorable las puntuaciones de 3.81 en adelante. Por otra parte, las puntuaciones que estaban entre $3.60 \mathrm{a}$ 3.80 se consideraron bien favorables y puntuaciones por debajo de los 3.59 se consideraron favorables. El promedio obtenido fue 3.65 considerado como una actitud bien favorable.

Como se mencionó, anteriormente, la puntuación más alta fue 3.87 con un $95.15 \%$ en desacuerdo con la premisa Considero que es una pérdida de tiempo discutir con el estudiante con impedimento sus necesidades de acomodo. Esto es cónsono con la bibliografía, la cual establece que como parte del proceso interactivo de acomodo razonable siempre se debe discutir con la persona que solicita el acomodo sus necesidades y cómo entiende que se puede compensar (Shaw, 2017). En la Tabla 2 se pueden observar otras de las premisas con menor variabilidad en las respuestas, donde las personas participantes demostraron una actitud sumamente favorable, estas son las premisas que obtuvieron un promedio de 3.81 o más.

Tabla 2: Distribución de actitudes sumamente favorables

3. Considero que es una pérdida de tiempo discutir con el estudiantado con impedimentos sus necesidades de acomodo

22. No le explico o describo la información en los documentos que entrego a estudiantes con impedimentos sensoriales (visuales o auditivos) porque se interrumpe la fluidez de la fila

7. No autorizo que el estudiantado con impedimento visual entre a la oficina con su perro guía ya que 3.84 puede distraer al resto del personal

21. Estoy dispuesto a que el estudiantado con impedimento utilice el equipo especializado que necesite 3.84 en la oficina.

30. Estoy dispuesto a garantizar al estudiantado con impedimentos igual derecho que al resto de estudiantes 3.84

29. Permitiría que estudiantes con impedimentos visuales o auditivos se sienten lo más cerca del área de 3.82 servicio

25. Tengo disposición para permitir la intervención de un ente lector o anotador al momento de brindar servicio a estudiantes con impedimento

Nota: Elaboración propia. 
http://doi.org/10.15359/ree.24-2.3

ROR: https://ror.org/01t466c14 Universidad Nacional, Costa Rica http://www.una.ac.cr/educare educare@una.cr

Por otra parte, hubo premisas que obtuvieron mayor variabilidad o dispersión en las respuestas. Un ejemplo lo es la premisa Después de discutir con el estudiante con impedimento sus necesidades de acomodo, realizo solo aquellos ajustes que sean pertinentes. Un $30.21 \%$ estuvo totalmente de acuerdoy un $32.29 \%$ de acuerdo, mientras que un $14.58 \%$ en desacuerdoy un 22.92 $\%$ totalmente en desacuerdo, para un promedio de 2.70; esta es la premisa con mayor variabilidad en sus respuestas y el promedio más bajo. Aunque no se exploró una posible explicación a esta variabilidad, puede estar relacionada con las preocupaciones y la falta de conocimiento sobre el tipo de acomodo apropiado. En relación con este aspecto, Schreuer y Sachs (2014) establecen que existe la necesidad de proveer mayor adiestramiento y conocimiento sobre cómo proveer y utilizar los acomodos de forma apropiada. Otro ejemplo de aseveraciones con variabilidad en respuestas es Estimo imprescindible que estudiantes con impedimentos se eduquen en instituciones universitarias especiales, que estén preparadas para atender sus necesidades educativas. El $48.54 \%$ estuvo en total desacuerdo. Un $29.13 \%$ expresó estar en desacuerdo, mientras que un $22.33 \%$ entre de acuerdo y totalmente de acuerdo. La Tabla 3 presenta otras premisas cuyo promedio es menor a los 3.60, considerado una actitud favorable.

Tabla 3: Distribución de actitudes favorables

\begin{tabular}{|c|c|}
\hline Premisa & Media \\
\hline $\begin{array}{l}\text { 31. Después de discutir con estudiantes con impedimento sus necesidades de acomodo, realizo solo } \\
\text { aquellos ajustes que sean pertinentes }\end{array}$ & 2.70 \\
\hline $\begin{array}{l}\text { 5. Estimo imprescindible que estudiantes con impedimentos se eduquen en instituciones universitarias } \\
\text { especiales, que estén preparadas para atender sus necesidades educativas. }\end{array}$ & 3.14 \\
\hline $\begin{array}{l}\text { 17. Pienso que estudiantes con impedimentos pueden prescindir de acomodos razonables en la oficina } \\
\text { y estar en igualdad de condiciones que el resto de estudiantes }\end{array}$ & 3.24 \\
\hline $\begin{array}{l}\text { 28. Pienso que realizar o hacer los acomodos o ajustes necesarios a estudiantes con impedimentos los } \\
\text { sitúan en mejor condición de aprendizaje y beneficios que el resto de estudiantes }\end{array}$ & 3.40 \\
\hline 15. Cuando realizo los ajustes o acomodos, estudiantes con impedimentos se beneficias más que el resto. & 3.42 \\
\hline $\begin{array}{l}\text { 10. Favorezco que se realicen acomodos para estudiantes con impedimentos, aunque altere la } \\
\text { planificación de los servicios que ofrezco. }\end{array}$ & 3.50 \\
\hline $\begin{array}{l}\text { 26. Pienso que estudiantes con impedimento pueden realizar los mismos trámites que el resto de } \\
\text { estudiantes sin necesidad de utilizar ningún tipo de modificación }\end{array}$ & 3.54 \\
\hline
\end{tabular}

Nota: Elaboración propia.

Por otra parte, en la Tabla 4 se pueden observar algunos ejemplos de las premisas que obtuvieron un promedio entre 3.61 a 3.80 . 
http://doi.org/10.15359/ree.24-2.3

ROR: https://ror.org/01t466c14 Universidad Nacional, Costa Rica

http://www.una.ac.cr/educare

educare@una.cr

Tabla 4: Distribución de actitudes bien favorables

Premisa

23. Accedo a que estudiantes cuyo impedimento les requiere llevar un anotador a la oficina, utilice este recurso porque pienso que lo necesita.

6. Si se asignaran estudiantes a mi área de trabajo permitiría que realicen los ajustes necesarios (acomodo razonable) para que puedan desempeñarse en su trabajo con igual posibilidad que el resto de estudiantes.

4. Si estudiantes con condición visual lo solicitan, aceptaría agrandar la letra en los documentos, para facilitar su acceso a la información.

1. Me resulta difícil aceptar la presencia de asistentes, lectores o intérpretes para sordos en la oficina, si estoy atendiendo a estudiantes con impedimentos.

9. Me siento incómodo cuando me accedo a realizar los ajustes necesarios (acomodo razonable) para que estudiantes con impedimento puedan beneficiarse al máximo del servicio que ofrezco.

8. Accedo a proveer el material del programa en impreso agrandado o en braille para que estudiantes con impedimentos visuales tengan accesibilidad a la información que distribuyo en la oficina.

12. Mantengo disposición a leer o explicar lo que dice en los documentos del programa, ya que es útil para estudiantes con impedimento que lo ameriten.
Media

3.78

3.76

3.75

3.75

Nota: Elaboración propia.

\section{Conclusión y recomendaciones}

El presente estudio tenía el propósito de explorar el conocimiento y las actitudes hacia los acomodos en el escenario universitario. Tanto estudiante, personal docente, y personal de otra índole de la universidad participaron. En general, los resultados reflejan falta de conocimiento, aun cuando se reconoce que al comparar los niveles de conocimiento con el estudio de Negrón y Suarez (2005), donde un 72 \% tuvo un conocimiento de moderado a bajo, alrededor de ese mismo 72 \% indicó en esta ocasión tener más conocimiento en las leyes relacionadas. No obstante, al explorar las implicaciones de los acomodos en los diversos escenarios, hubo entre las personas participantes, quienes aún los visualizan como ventajas para las personas que los reciben y no proveer la oportunidad de obtener igualdad de condiciones. A su vez, esto se refleja en la sección de actitudes, donde, por ejemplo, las personas participantes expresaron mayor variabilidad en las respuestas hacia la premisa Pienso que realizar o hacer los acomodos o ajustes necesarios a estudiantes con impedimentos les sitúan en mejor condición de aprendizaje y beneficios que el resto de estudiantes. 
http://doi.org/10.15359/ree.24-2.3

ROR: https://ror.org/01t466c14 Universidad Nacional, Costa Rica http://www.una.ac.cr/educare

Ahora bien, sería recomendable preguntar qué tipo de conocimiento es necesario. En general, en las investigaciones se ha encontrado necesidad de adquirir conocimiento relacionado con las necesidades particulares de las personas con diversidad funcional (Schreuer y Sachs, 2014; Zhang et al., 2010). En un estudio llevado a cabo por Basilice (2015) se encontró que las personas participantes expresaron un fuerte deseo y disposición de recibir adiestramiento profesional, con el fin de poder comprender mejor las necesidades de sus estudiantes con diversidad funcional. Esto significa que el adiestramiento no debe limitarse a dar a conocer las legislaciones vigentes, sino también, las necesidades particulares de estudiantes con diversidad funcional.

Por su parte, las personas participantes del estudio expresaron tener una actitud bien favorable hacia los acomodos. Esto sugiere que a mayor conocimiento mayor disposición para honrar los acomodos. Sin embargo, en estudios de actitudes hay una variable moderadora que no podemos dejar de considerar: el factor de deseabilidad social. El hecho de contestar lo que es correcto no necesariamente sugiere que esta es la forma en que realmente pienso, siento y actúo sobre el tema en cuestión. Sin embargo, el hecho de que el estudio fuera voluntario podría ser un indicador de que quienes participaron en efecto contestaron los cuestionarios con intención. No obstante, al explorar actitudes, una de las premisas que obtuvo mayor variabilidad en las respuestas fue: Estimo imprescindible que estudiantes con impedimentos se eduquen en instituciones universitarias especiales, que estén preparadas para atender sus necesidades educativas, escenario que estaría presentando condiciones de segregación y no inclusión, como se aspira a través de las distintas piezas legislativas.

Con losaños, la lucha por la inclusión de las personas con diversidad funcional en la sociedad se ha convertido en un asunto político y social. La creación de política pública ha protagonizado los esfuerzos para lograr esta inclusión. El derecho al acomodo razonable representa una herramienta de mucho valor. No obstante, aun con la existencia de distintas piezas legislativas que protegen los derechos de la población de personas con diversidad funcional, todavía prevalece el desconocimiento y la confusión sobre lo que implica este derecho al acomodo razonable. Es claro que la universidad tiene la responsabilidad social con el desarrollo y cambio de un país. Meléndez Alicea et al. (2012) establecen que esa responsabilidad social se refiere a:

La filosofía de gestión universitaria que pretende renovar el compromiso social de la universidad al mismo tiempo que facilita soluciones innovadoras a los retos que enfrenta la educación superior en el contexto de un mundo globalizado con un desarrollo más humano y sostenible. (p. 145)

Esto implica que debe contribuir al desarrollo del capital humano del futuro, sea este una representación de una persona con diversidad funcional o no. De esta forma estará contribuyendo al desarrollo de su potencial, inserción social y promoción de su calidad de vida. La educación y sensibilización han demostrado ser importantes, mas no suficientes. 
http://doi.org/10.15359/ree.24-2.3

ROR: https://ror.org/01t466c14 Universidad Nacional, Costa Rica

http://www.una.ac.cr/educare

educare@una.cr

A estas alternativas propongo la promoción de un desarrollo individual y sistémico sostenible. Un desarrollo sostenible implica planificar ahora sin sacrificar el mañana donde podamos atender las múltiples necesidades y estilos de aprendizaje y ejecución del personal y estudiantes con diversidad funcional dentro de los escenarios universitarios. La propuesta específica consiste en planificar tomando en consideración el diseño universal. El diseño universal implica el reconocimiento de la diversidad humana, la anticipación de una variedad de necesidades y un enfoque intencional para diseñar un entorno inclusivo (Shraeur y Sachs, 2014). Se basa en la filosofía de diseño de productos y ambientes que puedan ser utilizados por todas las personas en la medida que sea posible. El diseño universal puede ser una alternativa para todos los grupos humanos. Puede llegar a eliminar la necesidad de algunas modificaciones o acomodos, aun cuando las transformaciones continuarán siendo importantes para aquellos casos en que las necesidades del individuo no pueden ser satisfechas de otra manera (Lazarus et al., 2009). No obstante, se debe trabajar con los currículos de los programas académicos para que estos puedan planificar para la diversidad con la que se trabajará en un salón de clases. Esta, junto a las otras estrategias deben ser consideradas en los escenarios académicos, pues atienden las necesidades presentes y futuras de una población que, de acuerdo a la OMS, va en aumento y en el 2050 se convertirá en la minoría mayoritaria del mundo (OMS, 2011).

En cuanto a recomendaciones para estudios futuros, entiendo que se debe realizar otro estudio con el fin de explorar las necesidades particulares de las personas con diversidad funcional versus los servicios recibidos en la universidad, para el cual se use la metodología mixta para profundizar en la problemática y neutralizar el factor de deseabilidad social. De igual forma, divulgar estos resultados con las comunidades de educación post secundaria y que estas cultiven el desarrollo de una mejor sociedad, promoviendo la inclusión y la igualdad.

\section{Referencias}

Acta de Rehabilitación (1973) P. L. 93 - 112.

Americans With Disabilities Act, as amended (ADA). (1990). Pub. L. No. 101-336, 104 Stat. 327.

Báez, E. (2014). Experiencia de un grupo deestudiantes líderes universitarios con impedimentos asociados a la educación post secundaria y factores/elementos que inciden en la retención estudiantil (Tesis de maestría). Universidad de Puerto Rico, Recinto de Río Piedras, Puerto Rico.

Basilice, L. J. (2015). Faculty knowledge and attitudes regarding students with disabilities in higher education (Disertación doctoral). Dowling College, United States.

Bolt, S. E., Decker, D. M., Loyd, M. y Morlock, L. (2011). Students' perceptions of accommodations in high school and college. Career Development for Exceptional Individuals, 34(3), 165-175. https://doi.org/10.1177/0885728811415098 
http://doi.org/10.15359/ree.24-2.3

ROR: https://ror.org/01t466c14 Universidad Nacional, Costa Rica http://www.una.ac.cr/educare educare@una.cr

Concepción, K. y Rodríguez, A. I. (1998). Actitud de un grupo de pofesores de la Universidad de Puerto Rico, Recinto de Río Piedras, hacia el acomodo razonable de estudiantes con impedimento visual (Tesis de maestría). Universidad de Puerto Rico, Recinto de Río Piedras, Puerto Rico.

Copeland, J., Chan, F., Bezyak, J. y Fraser, R.T. (2010). Assessing cognitive and affective reactions of employers toward people with disabilities in the workplace. Journal of Occupational Rehabilitation, 20(4),427-434. https://doi.org/10.1007/s10926-009-9207-y

Erickson, W., Lee, C. y von Schrader, S. (2018). 2016 Disability status report: Puerto Rico. Ithaca, NY: Cornell University Yang-Tan Institute on Employment and disability (YTI). http://www. disabilitystatistics.org

Gold, P. B., Oire, S. N., Fabian, E. y Wewiorski, N. (2012). Negotiating workplace accommodations: Perceptions of employees with disabilities, employers, and rehabilitation service providers. Journal of Vocational Rehabilitation, 37(1), 25-37. https://doi.org/10.3233/JVR-2012-0597

Hernandez, B., Keys, C. y Balcazar, F. (2000). Employer attitudes toward workers with disabilities and their ADA employment rights: A literature review. Journal of Rehabilitation, 66(4), 4-16.

Innis-Johnson, J. E. (2012). The relationship of rehabilitation counselors' knowledge of the american with disabilities act 1990, attitudes toward reasonable accommodation, and job efficacy (Disertación doctoral). Wayne State University, United Sates, Michigan. https:// digitalcommons.wayne.edu/cgi/viewcontent.cgi? referer=\&httpsredir $=1$ \&article $=1445 \& c$ ontext=oa dissertations

Lazarus, S. S., Thurlow, M. L., Lail, K. E. y Christensen, L. (2009). A longitudinal analysis of state accommodations policies: Twelve years of change, 1993-2005. The Journal of Special Education, 43(2), 67-80. https://doi.org/10.1177/0022466907313524

Ley 44 (1985). Ley que prohíbe el discrimen contra las personas con impedimentos. https:// www.trabajo.pr.gov/docs/Unidad Antidiscrimen/Ley 44 Discrimen Impedidos.pdf

Ley 51 (7 de junio de 1996). Servicios educativos integrales para personas con impedimentos. http://www.lexjuris.com/lexlex/lexcodigoc/lexedimpedimentos.htm

Ley 238 (2004). Ley de la Carta de derechos de las personas con Impedimentos. http://www. lexjuris.com/lexlex/leyes2004/lexl2004238.htm

López, J. R., Correa, H. I., González, J. L. y Acevedo, W. I. (2016). Encuesta especial: Personas con impedimento en Puerto Rico marzo 2014. https://estadisticas.pr/files/Inventario/publicaciones/ DTRH ENCUESTADEPERSONASCONIMPEDIMENTOENPUERTORICO 2014 0.pdf 
http://doi.org/10.15359/ree.24-2.3

ROR: https://ror.org/01t466c14 Universidad Nacional, Costa Rica

http://www.una.ac.cr/educare

educare@una.cr

Meléndez Alicea, J., Torres Rivera, M. y Pacheco Sepúlveda, C. (2012). La responsabilidad social universitaria en Puerto Rico. En J. Domínguez Granda y C. Rama (Eds.), La responsabilidad social en la educación a distancia (pp. 145-157). Chimbote, Perú:Virtual Educa \& Universidad Católica Los Ángeles. https://virtualeduca.org/.../la responsabilidad social de la educacion a distancia.pdf

Negrón, C. y Suárez, M. (2005). Actitud de un grupo de profesores de la Universidad de Puerto Rico, Recinto de Cayey hacia el acomodo razonable de estudiantes con impedimentos (Tesis de maestría). Universidad de Puerto Rico, Recinto de Río Piedras, Puerto Rico.

Oliveras, L. E. (2012). Actitud y conocimiento hacia el acomodo razonable a estudiantes con impedimentos de un grupo de personal no docente que provee servicio directo a estudiantes de la Universidad de Puerto Rico, Recinto de Río Piedras (Tesis de maestría). Universidad de Puerto Rico, Recinto de Río Piedras, Puerto Rico.

Organización Mundial de la Salud (OMS). (2011). Informe mundial sobre la discapacidad: Resumen. Ginebra, Suiza: Autor. http://www.who.int/disabilities/world report/2011/ summary es.pdf?ua $=1$

Rao, S. y Gartin, B. C. (2003). Attitudes of university faculty toward accommodations to students with disabilities. Journal for Vocational Special Needs Education, 25(2-3), 47-54. https://eric. ed.gov/?id=EJ854908

Rojas-Rojas, L. M., Arboleda-Toro, N. y Pinzón-Jaime, L. J. (2018). Caracterización de población con discapacidad visual, auditiva, de habla y motora para su vinculación a programas de pregrado a distancia de una universidad de Colombia. Revista Electrónica Educare, 22(1), 1-28. https://doi.org/10.15359/ree.22-1.6

Saleh, M.C.y Bruyère. S.M. (2018). Leveraging employer practices in global regulatory frameworks to improve employment outcomes for people with disabilities. Social Inclusion, 6(1), 18-28. https://doi.org/10.17645/si.v6i1.1201

Schreuer, N. y Sachs, D. (2014). Efficacy of accommodations for students with disabilities in higher education. Journal of Vocational Rehabilitation, 40(1), 27-40. https://doi.org/10.3233/JVR$\underline{130665}$

Schwartz, A. (2016).Anational mixed methods research study:Defining reasonableaccommodations in higher education for adults with disabilities (Disertación doctoral). Walden University, Minneapolis, Minnesota, Estados Unidos. https://www.semanticscholar.org/paper/ANational-Mixed-Methods-Research-Study\%3A-Defining-Schwartz/70bce19c60cb6ed6c8 $\underline{\text { 89193454c6c9aa76f11605 }}$ 
http://doi.org/10.15359/ree.24-2.3

ROR: https://ror.org/01t466c14 Universidad Nacional, Costa Rica

Shaw, R. (2017). The disabled workforce: What the ADA never anticipated. A practical guide to help employers manage the growing requests for workplace accommodations. CreateSpace Independent Publishing Platform.

Timmerman, L. C. y Mulvihill, T. M. (2015). Accommodations in the college setting: The perspective of students living with disabilities. The Qualitative Report, 20(10), 1607-1625. http://nsuworks.nova.edu/tar/vol20/iss10/5

Unger, D. D. (2002). Employers' attitudes toward persons with disabilities in the workforce: Myths or realities? Focus on Autism and Developmental Disabilities, 17(1), 2-10. https://doi. org/10.1177/108835760201700101

Universidad de Puerto Rico Recinto de Río Piedras, Oficina de Planificación Académica. (2017). Estudiantes con impedimentos por año académico 2008-2017. Autor.

Zhang, D., Landmark, L., Reber, A., Hsu, H. Y, Kwok, O. y Benz, M. (2010). University faculty knowledge, beliefs, and practices in providing reasonable accommodations to students with disabilities. Remedial and Special Education, 31(4), 276-286. https://doi. org/10.1177/0741932509338348 